\title{
Association of hypocalcemia with in-hospital mortality and complications in patients with acute pulmonary embolism: results from the 2017 Nationwide Inpatient Sample
}

\author{
Mukunthan Murthi ${ }^{*}$, Hafeez Shaka ${ }^{1}$, Zain El-amir², Sujitha Velagapudi', Abdul Jamil ${ }^{2}$, Farah Wani ${ }^{3}$, \\ Ramtej Atluri', Akshay Kumar ${ }^{4}$ and Asim Kichloo ${ }^{3}$
}

\begin{abstract}
Background: Acute pulmonary embolism (PE) is a common cause for hospitalization associated with significant mortality and morbidity. Disorders of calcium metabolism are a frequently encountered medical problem. The effect of hypocalcemia is not well defined on the outcomes of patients with PE. We aimed to identify the prognostic value of hypocalcemia in hospitalized PE patients utilizing the 2017 Nationwide Inpatient Sample (NIS).

Methods: In this retrospective study, we selected patients with a primary diagnosis of Acute PE using ICD 10 codes. They were further stratified based on the presence of hypocalcemia. We primarily aimed to compare in-hospital mortality for PE patients with and without hypocalcemia. We performed multivariate logistic regression analysis to adjust for potential confounders. We also used propensity-matched cohort of patients to compare mortality.

Results: In the 2017 NIS, 187,989 patients had a principal diagnosis of acute PE. Among the above study group, 1565 $(0.8 \%)$ had an additional diagnosis of hypocalcemia. $12.4 \%$ of PE patients with hypocalcemia died in the hospital in comparison to $2.95 \%$ without hypocalcemia. On multivariate regression analysis, PE and hypocalcemia patients had 4 times higher odds (aOR-4.03, 95\% Cl 2.78-5.84, $\mathrm{p}<0.001$ ) of in-hospital mortality compared to those with only PE. We observed a similarly high odds of mortality $(a O R=4.4)$ on $1: 1$ propensity-matched analysis. The incidence of acute kidney injury (aOR $=2.62, \mathrm{Cl} 1.95-3.52, \mathrm{p}<0.001)$, acute respiratory failure $(\mathrm{aO} R=1.84, \mathrm{Cl} 1.42-2.38, \mathrm{p}<0.001)$, sepsis $(a O R=4.99, \mathrm{Cl} 3.08-8.11, \mathrm{p}<0.001)$ and arrhythmias $(\mathrm{aOR}=2.63, \mathrm{Cl} 1.99-3.48, \mathrm{p}<0.001)$ were also higher for $\mathrm{PE}$ patients with hypocalcemia.
\end{abstract}

Conclusion: PE patients with hypocalcemia have higher in-hospital mortality than those without hypocalcemia. The in-hospital complications were also higher, along with longer length of stay.

Keywords: Hypocalcemia, Pulmonary embolism, Mortality

*Correspondence: mukunthan338@gmail.com

1 John H Stroger Hospital of Cook County, 1969 W Ogden Ave, Chicago, IL 60612, USA

Full list of author information is available at the end of the article

\section{Background}

Pulmonary embolism (PE) is a common medical condition associated with significant morbidity and mortality. Although the exact incidence of PE is not known, approximately 900,000 persons are affected by DVT/PE per year in the United States. It is associated with significant mortality and morbidity, with sudden death being the

(C) The Author(s) 2021. Open Access This article is licensed under a Creative Commons Attribution 4.0 International License, which permits use, sharing, adaptation, distribution and reproduction in any medium or format, as long as you give appropriate credit to the original author(s) and the source, provide a link to the Creative Commons licence, and indicate if changes were made. The images or other third party material in this article are included in the article's Creative Commons licence, unless indicated otherwise in a credit line to the material. If material is not included in the article's Creative Commons licence and your intended use is not permitted by statutory regulation or exceeds the permitted use, you will need to obtain permission directly from the copyright holder. To view a copy of this licence, visit http://creativecommons.org/licenses/by/4.0/. The Creative Commons Public Domain Dedication waiver (http://creativecommons.org/publicdomain/zero/1.0/) applies to the data made available in this article, unless otherwise stated in a credit line to the data. 
first symptom in $25 \%$ of the patients [1]. Calcium plays a crucial role in multiple steps of the coagulation cascade including platelet adhesion, protease complex assembly and enzyme activation [2]. Hypocalcemia is associated with the progression of and poorer prognosis of numerous cardiopulmonary diseases [3, 4]. Calcium also is a part of the coagulation factor IV and thus is involved in the coagulation process [5].

Various factors are known to affect the outcomes of PE. One such factor being hypocalcemia.

Previously, it was suspected to be a potential reason for PE by inducing lower extremity spasms [6]. Validated prognostic scores like the Pulmonary Embolism Severity Index are currently widely used to determine mortality and morbidity in patients with PE [5]. However, there is no evidence defining the effect of hypocalcemia on the outcomes of patients hospitalized with PE. We aimed to identify the prognostic value of hypocalcemia in hospitalized PE patients utilizing the 2017 Nationwide Inpatient Sample.

\section{Methods}

We utilized the Nationwide Inpatient Sample (NIS) database of 2017 to perform this retrospective study. The NIS is the largest publicly available all-payer inpatient healthcare database in the United States. It approximates a $20 \%$ stratified sample of discharges from community hospitals and provides an estimate of inpatient healthcare utilization, charges, and outcomes. It estimates more than 35 million hospitalizations every year. The data available in NIS includes primary and secondary diagnosis during hospitalization, demographic characteristics, inpatient procedures, hospital charges, and overall outcome. We utilized ICD-10 codes to identify the diagnosis. Our study did not require Institutional review board (IRB) approval since NIS data is de-identified. We selected patients with a primary diagnosis of Acute Pulmonary embolism (PE) using the ICD-10 code. They were further stratified based on the presence of hypocalcemia using ICD-10 codes. ICD-10 codes I2692, I2693, I2694, I2694, I2699, I2602, I2609 were used to identify PE and E8351 was used to identify hypocalcemia diagnosis in the NIS database.

We primarily aimed to compare in-hospital mortality for PE patients with and without hypocalcemia. Secondary outcomes included the effect of hypocalcemia on the incidence of acute kidney injury (AKI), Acute respiratory failure (ARF), arrhythmias, sepsis, length of hospital stay (LOS) and total hospital charges (THC).

We used STATA ${ }^{\circledR}$ (StataCorp, College Station, TX) version 16.1 to perform statistical analysis. Healthcare Cost and Utilization Project (HCUP) supplies year-based discharge weights to calculate weighted national estimates.
Proportions were compared using Fisher's exact test. Independent sample t-test was used to compare the mean of continuous data. Univariate regression analysis was used to calculate the unadjusted odds ratio (OR) for each outcome. Based on the univariate screen significance $(\mathrm{p}<0.2)$, we selected variables to perform a multivariate logistic regression analysis to adjust for potential confounders. The multivariate regression model also included variables that were considered essential based on a literary review. The final multivariate model was adjusted for the following variables:: Age, race, COPD, Hypertension, Diabetes, Chronic Kidney disease, history of stroke, heart failure, history of venous thromboembolism, Charlson comorbidity index, hospital location/ teaching status, hospital bed size, dyslipidemia, zip-code wise median income status. Logistic regression was used for binary outcomes and linear regression for continuous outcomes. All p values were two-tailed, and we used a threshold of 0.05 to determine significance. Using the Charlson comorbidity index, we categorized patients into four groups based on $0,1,2$, and $\geq 3$ comorbidities.

We used propensity scores to match patients with PE who had hypocalcemia to those who did not. The resultant model had a 1:1 match of similar groups. The covariates used for propensity score calculation was age, race, COPD, chronic kidney disease, history of venous thromboembolism, dyslipidemia, heart failure, Charlson comorbidity index, hospital location/teaching status, hospital bed size, zip-code wise median income status. For matching cases and controls in propensity analysis, a double robust method was initially used to generate treatment weights. Inverse probability of treatment weighting was used to match cases to controls using generalized linear models. The propensity model included 1565 cases and controls. The propensity-matched cohort of patients was then used to compare the mortality.

\section{Results}

Among all patient encounters in the 2017 NIS, 187,989 had a principal diagnosis of acute pulmonary embolism. Among them, 1565 (0.8\%) had an additional diagnosis of hypocalcemia (Fig. 1). The mean age of PE patients with and without hypocalcemia was 61.7 and 62.6 years respectively $(\mathrm{p}<0.001)$. There was no significant difference in the racial distribution of patients between the two groups $(\mathrm{p}=0.062)$. Patients with hypocalcemia had a higher number of comorbid conditions (Charlson index $\geq 3-39.9 \%$ vs. $28 \%$ ) than without hypocalcemia (Table 1). The study did not show any statistical difference in the percentage of males, mean age, race, hospital location, and bed size. The insurance types for patients with and without hypocalcemia was not significantly different $(p=0.656)$ with Medicare being the most common 


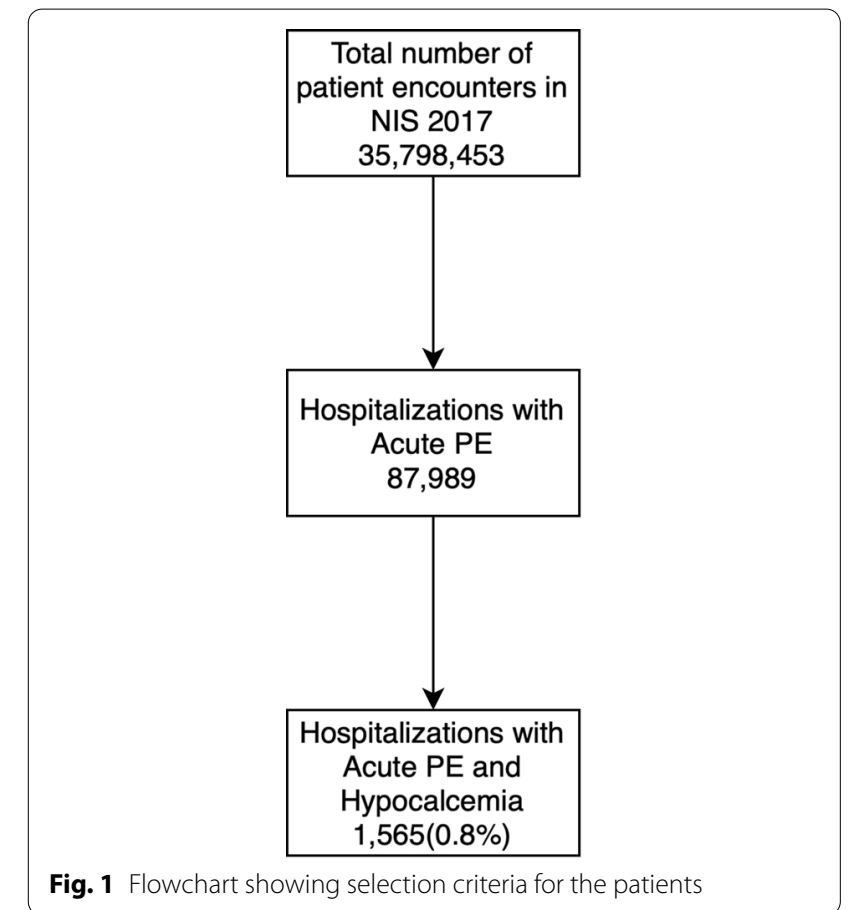

insurance type in both groups (51.8\% vs $52 \%) .12 .4 \%$ of $\mathrm{PE}$ patients with hypocalcemia died in hospital, compared to $2.95 \%$ in those without hypocalcemia. On multivariate regression analysis adjusting for confounders, $\mathrm{PE}$ and hypocalcemia patients had 4 times higher odds (aOR-4.03, 95\% CI 2.78-5.84, $\mathrm{p}<0.001$ ) of in-hospital mortality compared to those with only PE.

On multivariate analysis, the LOS was longer for patients with hypocalcemia (7.41 vs 4.26 days), with an adjusted increase in LOS by 2.8 days $(\mathrm{p}=<0.001)$ (Table 2). The incidence of acute kidney injury $(\mathrm{aOR}=2.62$, CI $1.95-3.52 \mathrm{p}<0.001)$, acute respiratory failure $(\mathrm{a} 0 \mathrm{R}=1.84, \mathrm{CI} 1.42-2.38 \mathrm{p}<0.001)$, sepsis $(\mathrm{aOR}=4.99$, CI $3.08-8.11 \mathrm{p}<0.001)$ and arrhythmias $(\mathrm{aOR}=2.63$, CI $1.99-3.48 \mathrm{p}<0.001)$ were also higher for PE patients with hypocalcemia. Using propensity matching patients with Hypocalcemia and PE had 4.4 times higher odds of in-hospital mortality $(\mathrm{aOR}=4.43, \mathrm{CI}$ 3.03-6.50, $\mathrm{p}<0.001$ ) compared to those with only PE.

\section{Discussion}

Our study demonstrated worse in-hospital mortality among PE patients with hypocalcemia than without hypocalcemia. The in-hospital complications were also higher, and hypocalcemia patients were hospitalized for 2.8 days longer on average. Although previous smaller studies have shown this association of hypocalcemia and
Table 1 Baseline characteristics of hospitalizations for PE with and without hypocalcemia

\begin{tabular}{|c|c|c|c|}
\hline & PE only, \% & $\begin{array}{l}\text { PE and } \\
\text { hypocalcemia, } \\
\%\end{array}$ & $p$-value \\
\hline Total no & 186,242 & 1565 & \\
\hline Mean age (years) & 62.6 & 61.7 & $<0.001$ \\
\hline Female & 51.9 & 50.8 & 0.67 \\
\hline Race & & & 0.062 \\
\hline White & 71.9 & 65.8 & \\
\hline Black & 18.9 & 22.1 & \\
\hline Hispanic & 5.5 & 5.9 & \\
\hline Asians or Pacific Islander & 0.9 & 2.34 & \\
\hline \multicolumn{4}{|l|}{ Comorbidities } \\
\hline Hypertension & 41.7 & 36.4 & 0.056 \\
\hline Diabetes & 22.3 & 23.3 & 0.70 \\
\hline CKD & 12.1 & 14.7 & 0.194 \\
\hline COPD & 17.4 & 18.5 & 0.611 \\
\hline Previous VTE & 16.7 & 12.1 & 0.036 \\
\hline Dyslipidemia & 35.5 & 33.5 & 0.486 \\
\hline Previous stroke & 1.6 & 0.9 & 0.331 \\
\hline Heart failure & 15.3 & 20.1 & 0.023 \\
\hline Charleston comorbidity index & & & $<0.001$ \\
\hline 0 & 32.1 & 18.8 & \\
\hline 1 & 23.3 & 18.8 & \\
\hline 2 & 16.4 & 22.3 & \\
\hline$\geq 3$ & 28 & 39.9 & \\
\hline Hospital type & & & 0.0009 \\
\hline Rural & 9.8 & 5.7 & \\
\hline Urban nonteaching & 23.8 & 17.8 & \\
\hline Urban teaching & 66.3 & 76.3 & \\
\hline Expected primary payer & & & 0.6562 \\
\hline Medicare & 51.8 & 52 & \\
\hline Medicaid & 12 & 13.7 & \\
\hline Private & 29.5 & 27.1 & \\
\hline Self-pay & 3.7 & 3.5 & \\
\hline $\begin{array}{l}\text { Median household income, in } \\
\text { US \$ }\end{array}$ & & & 0.028 \\
\hline $1-43,999$ & 28.3 & 33.9 & \\
\hline $44,000-55,999$ & 27.2 & 24.9 & \\
\hline $56,000-73,999$ & 24.4 & 26.5 & \\
\hline $74,000+$ & 19.9 & 14.5 & \\
\hline
\end{tabular}

PE, our study is the largest and the first performed using a population database to corroborate these findings.

Calcium is involved in platelet function and numerous parts of the coagulation cascade [7]. Its role in the coagulation cascade may partly explain the association of hypocalcemia with such outcomes. Calcium may also result in arterial relaxation and reduced blood pressure through perivascular receptor activation, leading 
Table 2 Multivariate regression analysis of primary and secondary outcomes

\begin{tabular}{lllll}
\hline & PE & PE and hypocalcemia & Adjusted odds ratio (aOR) & p-value \\
\hline $\begin{array}{l}\text { Primary outcome } \\
\text { Death }\end{array}$ & 2.95 & 12.4 & $4.03(2.78-5.84)$ & $<0.001$ \\
Secondary outcomes & & & & $<0.001$ \\
AKI & 11 & 24 & $2.62(1.95-3.52)$ & $<0.001$ \\
ARF & 22 & 35 & $1.84(1.42-2.38)$ & $<0.001$ \\
Sepsis & 1.2 & 6.7 & $4.99(3.08-8.11)$ & $<0.001$ \\
Arrhythmias & 16 & 30 & $2.63(1.99-3.48)$ & \\
\hline
\end{tabular}

Multivariate analysis adjusted for the following variables: Age, race, COPD, HTN, DM, CKD, history of stroke, heart failure, history of venous thromboembolism, Charlson comorbidity index, hospital location/teaching status, hospital bed size, dyslipidemia, zip-code wise median income status

to hematoma expansion in bleeding patients. Morotti et al. reported such an association in patients with intracerebral hemorrhage [8].

Patients with hypocalcemia had a higher number of comorbid conditions (Charlson index $\geq 3-39.9 \%$ vs $28 \%$ ). One possible explanation could be the higher prevalence of renal dysfunction in such patients predisposing them to low calcium levels [9]. The incidence of acute kidney injury, acute respiratory failure, sepsis, and arrhythmias were higher for PE patients with hypocalcemia. The increased incidence of the above conditions may be partly due to more comorbid conditions in patients with hypocalcemia. Additionally, hypocalcemia itself has been linked to certain complications. Severe hypocalcemia has been linked to life-threatening cardiac arrhythmia and seizures [10]. Low calcium levels have been specifically discussed in relation to several neurological and cardiac complications, including seizures, status epilepticus, and coma among other complications, although there is discussion as to whether or not these complications may be explained by other causes unrelated to calcium [10]. Moreover, in patients hospitalized with COVID-19 infection, hypocalcemia has been found to be associated with increased risk of acute respiratory failure and mortality, underscoring the risk of more complicated hospitalization courses [11].

Previous studies have identified hypocalcemia as an independent predictor of mortality after acute pulmonary thromboembolism. Reports have indicated that pulmonary thromboembolism patients with hypocalcemia had higher death rates than those without hypocalcemia [5]. Additionally, there is an association between hypocalcemia and increased mortality in critically ill patients [12]. In critically ill patients, hypocalcemia is thought to occur most often because of heart failure and hyperadrenergic states [13]. The mechanism of calcium disequilibrium in critically ill patients may be due to end-organ resistance to parathyroid hormone, impairment of the parathyroid hormone secondary to catecholamine excess or pro-inflammatory cytokine release and inhibited parathyroid hormone release and cellular redistribution of calcium. Vitamin D deficiency or insufficiency may also be present in critically ill patients and is reported to be prevalent in as many as $78 \%$ of intensive care unit (ICU) patients [13]. Patients with PE may require ICU hospitalizations and may require mechanical ventilation, catheterization, and thrombolytic use [14]. These results warrant further studies to confirm these findings and investigate possible mechanisms.

The presence of increased mortality in patients with hypocalcemia may suggest that hypocalcemia may be a useful prognostic tool in PE patients. There are several prognostic assessment tools that exist for PE patients, including the pulmonary embolism severity index (PESI) and the simplified PESI (sPESI) $[15,16]$. Moreover, imaging and cardiac biomarkers are also used to stratify patients with an intermediate and high risk of PE [17]. Previous research has identified hypocalcemia to be an independent predictor of 30-day mortality following a PE. An optimal prediction rule, which contains hypocalcemia and several variables from the PESI and sPESI, has been shown to have higher predictive value and validity than the PESI and sPESI respectively [5]. Our findings endorse the relationship between hypocalcemia and in-hospital mortality that has been discussed in the literature, and hypocalcemia may be a valuable future prognostic tool for PE patients.

\section{Strengths and limitations}

One can appreciate several limitations in this study. This study examines numerous demographics of PE hospitalizations, offering a comprehensive and thorough overview of PE hospitalizations. We obtained the population used for this study from the NIS, an extensive, multiethnic hospital-based registry. However, as with any study, there are limitations that should be noted. Data from the NIS is subject to biases associated with retrospective studies. Additionally, the 
NIS reports information on hospitalizations and not from individual patients; therefore, patients admitted numerous times would be included more than once in the data set. The database also uses ICD-10 codes to report information, leaving it subject to coding errors. Thus, hypocalcemia itself may not have been coded in every patient. Additionally, the exact etiology of hypocalcemia cannot be elicited through NIS. Finally, the NIS does not include information about the severity of the diagnosis at the time of admission. For example, it does not include the precise calcium level of patients with hypocalcemia.

Despite the limitations mentioned above, the large sample size, outcomes of the study, and analysis techniques make for a study that provides a comprehensive overview of hypocalcemia and PE while aiming to encourage further discourse and future controlled prospective studies.

\section{Conclusion}

Pulmonary embolism (PE) is associated with significant mortality and morbidity, and disorders of calcium metabolism are a commonly encountered medical problem. Our study showed that patients with PE and hypocalcemia had higher in-hospital mortality compared to those without hypocalcemia. These patients also had more comorbid conditions, as reflected by the Charlson comorbidity index. Patients hospitalized with a $\mathrm{PE}$ and concurrent hypocalcemia may be at higher risk of numerous in-hospital complications, including sepsis, arrhythmias, acute kidney injury, and death. The findings of our study may reflect the effectiveness of using calcium as a prognostic marker in patients hospitalized with a pulmonary embolism.

\section{Abbreviations \\ NIS: Nationwide Inpatient Sample; HCUP: Healthcare Cost and Utilization Pro- ject; PE: Pulmonary embolism; AKI: Acute kidney injury; ARF: Acute respiratory failure; LOS: Length of hospital stay; THC: Total hospital charges.}

\section{Acknowledgements}

Not applicable.

\section{Authors' contributions}

MM, HS, RA analyzed and interpreted the database. SV, MM, ZE, AJ, FW, AK, AK contributed the manuscript. All authors read and approved the final manuscript.

\section{Funding}

None.

\section{Availability of data and materials}

The datasets generated and/or analysed during the current study are available in the Healthcare Cost and Utilization Project repository, https://www.hcup-us. ahrq.gov/nisoverview.jsp

\section{Declarations}

Ethics approval and consent to participate

Not applicable.

\section{Consent for publication}

Not applicable.

\section{Competing interests}

The authors declare that they have no competing interests.

\section{Author details}

1 John H Stroger Hospital of Cook County, 1969 W Ogden Ave, Chicago, IL 60612, USA. ² Department of Internal Medicine, Central Michigan University College of Medicine, Saginaw, MI, USA. ${ }^{3}$ Department of Internal Medicine, Samaritan Medical Center, Watertown, NY, USA. ${ }^{4}$ Department of Cardiothoracic Surgery, University of Pittsburgh Medical Centre, Pittsburgh, USA.

Received: 21 August 2021 Accepted: 9 December 2021

Published online: 11 December 2021

\section{References}

1. Beckman MG, Hooper WC, Critchley SE, Ortel TL. Venous thromboembolism: a public health concern. Am J Prev Med. 2010;38(4 Suppl):S495-501.

2. Mikaelsson ME. The role of calcium in coagulation and anticoagulation. In: Sibinga CTS, Das PC, Mannucci PM, editors. Coagulation and blood transfusion developments in hematology and immunology, vol. 26. Boston: Springer; 1991.

3. Miura S, Yoshihisa A, Takiguchi M, Shimizu T, Nakamura Y, Yamauchi $\mathrm{H}$, et al. Association of hypocalcemia with mortality in hospitalized patients with heart failure and chronic kidney disease. J Card Fail. 2015;21(8):621-7.

4. Yamaguchi S, Hamano T, Doi Y, Oka T, Kajimoto S, Kubota K, et al. Hidden hypocalcemia as a risk factor for cardiovascular events and all-cause mortality among patients undergoing incident hemodialysis. Sci Rep. 2020;10(1):4418.

5. Wang X, Xiang Y, Zhang T, Yang Y, Sun X, Shi J. Association between serum calcium and prognosis in patients with acute pulmonary embolism and the optimization of pulmonary embolism severity index. Respir Res. 2020;21(1):298.

6. Usta B, Gozdemir M, Turgut F, Sert H, Kanbay M, Demircioglu Rl, et al. Does hypocalcemia trigger pulmonary embolism? Med Hypotheses. 2007;68:464-5.

7. Palta S, Saroa R, Palta A. Overview of the coagulation system. Indian J Anaesth. 2014;58(5):515-23.

8. Morotti A, Charidimou A, Phuah C-L, Jessel MJ, Schwab K, Ayres AM, et al. Association between serum calcium level and extent of bleeding in patients with intracerebral hemorrhage. JAMA Neurol. 2016;73(11):1285-90.

9. Hill Gallant KM, Spiegel DM. Calcium balance in chronic kidney disease. Curr Osteoporos Rep. 2017;15(3):214-21.

10. Duval M, Bach K, Masson D, Guimard C, Le Conte P, Trewick D. Is severe hypocalcemia immediately life-threatening? Endocr Connect. 2018;7(10):1067-74.

11. di Filippo L, Formenti AM, Doga M, Frara S, Rovere-Querini P, Bosi E, et al. Hypocalcemia is a distinctive biochemical feature of hospitalized COVID19 patients. Endocrine. 2021;71(1):9-13.

12. Liu J, Han P, Wu J, Gong J, Tian D. Prevalence and predictive value of hypocalcemia in severe COVID-19 patients. J Infect Public Health. 2020;13(9):1224-8.

13. Zhang Z, Xu X, Ni H, Deng H. Predictive value of ionized calcium in critically ill patients: an analysis of a large clinical database MIMIC II. PLOS ONE. 2014;9(4):e95204.

14. Admon AJ, Seymour CW, Gershengorn HB, Wunsch H, Cooke CR. Hospital-level variation in ICU admission and critical care procedures for patients hospitalized for pulmonary embolism. Chest. 2014;146(6):1452-61. 
15. Dentali F, Riva N, Turato S, Grazioli S, Squizzato A, Steidl L, et al. Pulmonary embolism severity index accurately predicts long-term mortality rate in patients hospitalized for acute pulmonary embolism. J Thromb Haemost. 2013;11(12):2103-10.

16. Wells P, Peacock WF, Fermann GJ, Coleman Cl, Wang L, Baser O, et al. The value of sPESI for risk stratification in patients with pulmonary embolism. J Thromb Thrombolysis. 2019;48(1):149-57.

17. Konstantinides SV, Meyer G, Becattini C, Bueno H, Geersing G-J, Harjola V-P, et al. 2019 ESC Guidelines for the diagnosis and management of acute pulmonary embolism developed in collaboration with the European Respiratory Society (ERS): The Task Force for the diagnosis and management of acute pulmonary embolism of the European Society of Cardiology (ESC). Eur Heart J. 2019;41(4):543-603.

\section{Publisher's Note}

Springer Nature remains neutral with regard to jurisdictional claims in published maps and institutional affiliations.
Ready to submit your research? Choose BMC and benefit from:

- fast, convenient online submission

- thorough peer review by experienced researchers in your field

- rapid publication on acceptance

- support for research data, including large and complex data types

- gold Open Access which fosters wider collaboration and increased citations

- maximum visibility for your research: over $100 \mathrm{M}$ website views per year

At BMC, research is always in progress.

Learn more biomedcentral.com/submissions 